\title{
Path Integrals and Supersolids
}

\author{
D. M. CEPERLEY* \\ Department of Physics, University of Illinois Urbana-Champaign, \\ Urbana, IL 61801, USA \\ *E-mail: ceperley@uiuc.edu
}

\begin{abstract}
Recent experiments by Kim and Chan on solid ${ }^{4}$ He have been interpreted as discovery of a supersolid phase of matter. Arguments based on wavefunctions have shown that such a phase exists, but do not necessarily apply to solid ${ }^{4} \mathrm{He}$. Imaginary time path integrals, implemented using Monte Carlo methods, provide a definitive answer; a clean system of solid ${ }^{4} \mathrm{He}$ should be a normal quantum solid, not one with superfluid properties. The Kim-Chan phenomena must be due to defects introduced when the solid is formed.
\end{abstract}

Keywords: Path Integral, Monte Carlo, superfluid, supersolid

\section{Introduction}

Around the year 1970, there were several papers suggesting that a quantum solid, such as ${ }^{4} \mathrm{He}$ could show superfluidity and bose-condensation. First, Andreev and Lifshitz ${ }^{1}$ introduced the vacancy model. Assuming that a quantum solid contains a non-zero fraction of mobile vacancies, it is inevitable that at sufficiently low temperature they will bose condense. The resulting system will have superfluid properties. Chester ${ }^{2}$ arrived at the same conclusion using a Jastrow (or pair product) wavefunction. Solids described by such a wavefunction must have vacancies (assuming the Jastrow wavefunction is short ranged), and a non-zero condensate fraction at zero temperature. We can then ask what type of interaction will have a Jastrow wavefunction as its ground state: the interaction will have a very strong three-body component; stronger than the pair interaction and very different from the interaction between helium atoms. The unanswered questions are then a) are there enough vacancies in ${ }^{4} \mathrm{He}$ at low temperature, b) is a Jastrow wavefunction adequate to describe a quantum solid, and c) are there any other mechanisms that can cause a quantum solid to be superfluid? 
In $2004 \mathrm{Kim}$ and $\mathrm{Chan}^{3}$ reported superfluid-like responses in their torsional oscillator (TO) experiments. In these experiments, the moment of rotational inertia of a small cylinder containing the solid helium sample is measured by monitoring its period. They found that below approximately $0.15 \mathrm{~K}$, a small fraction (up to $2 \%$ ) of the helium no longer oscillates with the cylinder, but is presumably at rest. This would be understood within the vacancy model as vacancies being at rest.

For a recent review of the experimental situation, please consult Balibar and Caupin ${ }^{4}$ and for the theory, Prokofev. ${ }^{5}$ This article does not present new results, nor is it an extensive review. In the context of this path integral conference proceedings, we sketch how path integrals lead to a deeper and unique understanding of the problem of supersolid ${ }^{4} \mathrm{He}$, an analysis not available with other methods.

\section{Path Integral Methods}

Imaginary time path integrals (ITPI) were introduced by Feynman ${ }^{6}$ to understand superfluidity of ${ }^{4} \mathrm{He}$, leading to the ring exchange model. ITPIs are an exact mapping of equilibrium statistical mechanics of quantum bosons onto the classical statistical mechanics of a peculiar "ring polymer" model. Let $R(t) \equiv\left\{\mathbf{r}_{1}(t), \mathbf{r}_{2}(t), \ldots \mathbf{r}_{N}(t)\right\}$ be the $3 \mathrm{~N}$ coordinates of $N$ helium atoms at imaginary time $t$. Then a path consists of a trajectory $R(t)$ where $0 \leq t \leq \beta$ and $\beta=1 / k_{B} T$ with $T$ the temperature, and, for computational reasons, we work with discrete time; imaginary time is in steps of $\tau$ so that $t=k \tau$ and $M=\beta / \tau$. The probability of a path is given by $\exp \left(-\sum_{k=1}^{M} S(R((k-1) \tau), R(k \tau))\right)$ where $S\left(R, R^{\prime}\right)$ is the link-action. In the so-called primitive approximation, this is simply the kinetic and potential action, while in actual implementation more accurate forms are used. See the review article ${ }^{9}$ for a detailed discussion of numerical methods.

What remains is to set the initial and final boundary conditions for the path. For calculations of scalar properties of bosons, one uses periodic boundary conditions in time, but allowing for exchange so that $R(0)=\hat{P} R(\beta)$ where $P$ is a specific permutation of particle labels, all $N$ ! permutations being allowed. Then, as shown by Feynman, ${ }^{6}$ the lambda transition in liquid ${ }^{4} \mathrm{He}$ occurs because the contributing permutations change from small exchange cycles above $T_{\lambda}$, to macroscopic exchanges below $T_{\lambda}$. There are two other important connections between quantum collective behavior and the ring polymer model, namely, the superfluid density and bose condensation.

The superfluid density is defined in terms of the free energy needed to 
place a sample in rotation, for an infinitesimally small angular velocity. ( $n b$ this is steady state rotation, not oscillation as in the TO experiment.) It is possible to show that the superfluid density fraction can be calculated within ITPI as ${ }^{7}$

$$
\frac{\rho_{s}}{\rho}=\frac{\left\langle\left(2 m A_{z}\right)^{2}\right\rangle}{\beta \hbar^{2} I}=\frac{m\left\langle W_{z}^{2}\right\rangle}{\beta \hbar^{2} N}
$$

where $A_{z}$ is the path area projected on the $x y$ plane, $I$ is the classical moment of inertia and $m$ the mass. For periodic boundary conditions, we use the second formula which contains the "winding number" of the path $W_{z}$ in the $z$ direction. In either case, in the thermodynamic limit, nonzero superfluid fraction can only come from path involving a macroscopic number of atoms forming a long exchange cycle.

The second property is the condensate fraction. Again, it can be shown ${ }^{8}$ that the fraction of atoms with precisely zero momentum, $n_{0}$, is given by the large distance limit of the single particle density matrix, $n(r)$, and can by calculated in ITPI by the probability that the ends of a cut "polymer" (i.e. one not constrained to be periodic in IT) are separated from each other by a large distance. Both of these properties have been demonstrated to work ${ }^{9}$ for liquid helium using the same potential as solid helium, but at a lower density.

Now turning to quantum crystals, the path picture of a quantum crystal is that of a "polymer" crystal; a set of $N$ such polymers in a periodic box that spontaneously order spatially. Experimentally, and in computer simulations, we define the solid phase by the presence of a Bragg peak in the structure factor, $S(k)$, that scales with the number of atoms. If the particles are bosons, then they are allowed to cross-link (form ring exchanges), however, they may choose not to. At low enough temperature, one will always find a few isolated local ring exchanges, e. g. a neighboring pair of atoms exchanging. Note that this exchange does not contribute to either the superfluid density or to the condensate fraction. It is not a winding exchange because it is local. As in superfluid helium, we need a winding exchange to signal supersolidity, which necessarily involves a macroscopic number of atoms.

Concerning the question of whether the ground state has vacancies, one is faced with a problem of how to define a vacancy in a quantum crystal. Because of quantum fluctuations, many lattice sites may be temporarily vacant, but if the there is a nearby interstitial atom, these are not really vacancies but rather vacancy-interstitial pairs (VIPs). The number of true vacancies equals the number of lattice sites minus the number of atoms; 
both can be experimentally measured. For calculations in the canonical ensemble, one adds or subtracts an atom from a perfect crystal, while changing the box size to keep the density the same, ${ }^{10}$ while in the grand canonical ensemble one looks at the slope of the single particle Green's function. ${ }^{11}$ Both approaches lead to a consistent value of the excitation energy of a vacancy of about $14 \mathrm{~K}$ and of an interstitial of $23 \mathrm{~K}$, for the lowest, most favorable density of solid ${ }^{4} \mathrm{He}$. Since the the energy for these point defects, is non-zero and large, we do not expect them to be present in solid ${ }^{4} \mathrm{He}$ at low temperatures, thus making the vacancy mechanism for supersolidity invalid. Questions have been raised ${ }^{13}$ whether there could be finite size effects that invalidates this conclusion.

ITPI uniquely put the questions of supersolidity in a clear light. Methods based on wavefunctions, such as the Chester proof of BEC, ${ }^{2}$ are only as valid as long as the wavefunction is correct. It is difficult to make the connection between the wavefunction and the Hamiltonian. Typically a wavefunction is justified on the basis of how well other properties, such as the ground state energy, compare to experiment. However, the important properties we have just mentioned, the condensate fraction and the superfluid density, are far off-diagonal properties of the density matrix, and very different from a local property such as the energy, so that such methods of establishing the accuracy of a wavefunction are not reliable. Let us discuss two qualitative arguments based on ITPI.

Suppose a quantum crystal is superfluid; then it must have a macroscopic winding exchange. One possibility is that all the atoms simply slide along a row in the crystal, however, this process has a very low probability because all the atoms would have to move simultaneously. A simple way for such a process to proceed is for a VIP to form, for the vacancy and interstitial to separate and then diffuse independently; if one or other of the defects winds around the boundary before recombining (as required by the periodicity in time), then the system is a supersolid. However, during this diffusion, it is clear that there must be isolated vacancies and interstitials, and if this process is frequent, then there is no reason that either vacancies or interstitials would not be stable by themselves. So Prokofev and Svistunov ${ }^{14}$ conclude that a stable point defects are both necessary and sufficient for a supersolid. The weak point of the argument is the possible existence of more complicated mechanisms leading to winding exchanges. We searched ${ }^{22}$ for the most probable mechanism for winding exchanges, and found that they occur in a "plasma" state of VIPs, however, such a state is not stable. 
A second ITPI argument ${ }^{18}$ shows that there does exist a normal phase for quantum crystals. On the contrary, it has been suggested that any bose system at low enough temperature will bose condense. Take a system heavier than helium that forms a good crystal, e.g. solid ${ }^{132} \mathrm{Xe}$. Because of its heavy mass, the atoms sit at the absolute minimum of the potential energy. If we imagine a winding path, needed to have a non-zero superfluid density, the winding path must necessarily have a higher potential energy. So in the limit of low temperature, this will have an action above the ground state action by an amount at least proportional to a macroscopic length, $N^{1 / 3}$. Thus in the $\beta \rightarrow \infty$ limit the extra action will scale to $\infty$ and such winding paths will not contribute to the partition function. Hence the ground state of ${ }^{132} \mathrm{Xe}$ has $\rho_{s}=0$. Solid helium is unusual in that at zero temperature, the lattice constant is larger than required to sit at the minimum of the potential, so this argument does not apply; it could lower its energy by exchanging.

\section{Path integral results}

The above discussion was meant to be general. The real utility of ITPI is to apply them on the actual Hamiltonian of many-body helium, since as we have seen there exist Hamiltonians that have a supersolid ground state, and those that have a normal solid ground state. Does solid ${ }^{4} \mathrm{He}$ fall into either of those categories? Now helium is unique in that a helium atom is well-represented as an elementary particle interacting in a pairwise fashion (in a restricted range of temperatures and densities) because the electronic gap is more than $2 \times 10^{5} \mathrm{~K}$. Semi-empirical potentials such as Aziz et al ${ }^{19}$ are correct to about $0.1 \%$; the errors concern the three- and higher body terms. Numerous comparisons with experiment bear out this point of view. For example, the Debye-Waller factors ${ }^{20}$ of solid helium (both isotopes) are in very good agreement (in some cases better than 1\%) with experiment for a wide range of density and pressure. We already discussed the results of the vacancy energy. PIMC is in agreement with some of the vacancy data, but the experimental results vary widely.

Two independent ITPIMC calculations ${ }^{11,15}$ for the single particle density matrix have been made. Both show the function decays exponentially fast (but with oscillations caused by the lattice), reaching a value of roughly $10^{-6}$ at the edge of the box $15 \AA$ distant. If it stabilized at this value it would be an incredibly small condensate fraction. We note that this result is different than computed using the shadow wave function technique, ${ }^{21}$ which gives stable condensate fraction of $5 \times 10^{-6}$. It seems clear that the finite value 
comes because the SWF has inherited the properties of the Jastrow wave function, and recent calculations on $2 \mathrm{D}$ solid helium confirm this view. ${ }^{21}$

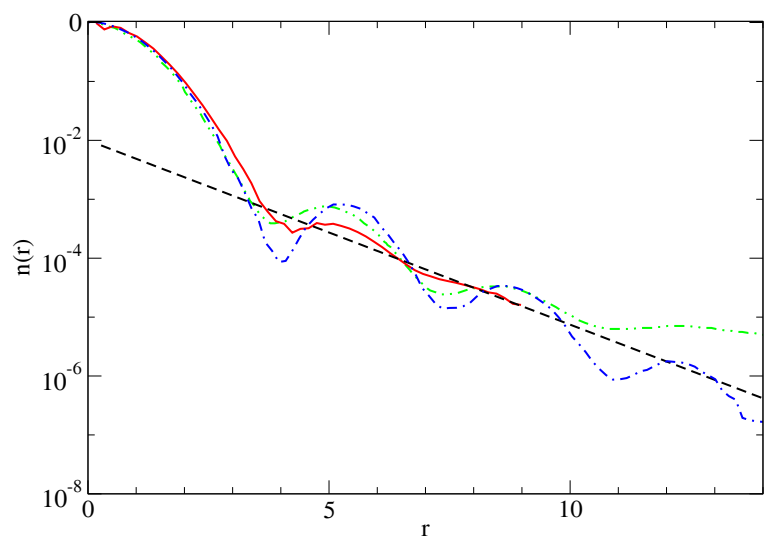

Fig. 1. (color online) The single particle density matrix ${ }^{15}$ as a function of distance (in $\AA$ ), estimated with PIMC, in hcp ${ }^{4} \mathrm{He}$ at a density $286 \mathrm{~nm}^{-3}$ and a temperature of $0.5 \mathrm{~K}$. The solid (red) curve is the spherically averaged $\mathrm{n}(\mathrm{r})$ while the dot-dashed (blue) curve is $n(x)$. The upper double-dot dashed (green) curve is a variational MC calculation using the shadow wave function ref. ${ }^{13}$ The dashed straight line (black) has a slope determined from the exchange calculations of ref. ${ }^{16}$

The PIMC results also do not show any winding exchanges and hence no superfluid. In order to understand the situation, we did an detailed analysis of the probability of a given ring exchange; it is these ring exchange frequencies ${ }^{17}$ that give rise to the magnetic ordering in solid ${ }^{3} \mathrm{He}$. At low temperatures, there are infrequent local exchanges, but the probability of long exchanges drops exponentially fast, thus solid ${ }^{4} \mathrm{He}$ is predicted to be a normal (insulating) quantum solid, not a supersolid. In conclusion, wellcontrolled numerical calculations predict that solid ${ }^{4} \mathrm{He}$ should not be a supersolid.

\section{Conclusion and Prospectives}

Because of the excitement over the Kim-Chan experiments, there have been numerous new experiments in the last few years. Several groups have reproduced the TO experiments. There have been several experiments that 
directly address the issue of phase transition. Lin et al ${ }^{23}$ have performed a high precision heat capacity measurement, and did not see any direct evidence of the lambda transition expected for the vacancy mechanism. At low temperature they did see a linear term, possibly due to the heat capacity of lower dimensional structures such as grain boundaries or dislocations. In scattering experiments, ${ }^{24}$ it was found that the Bragg peak persisted to low temperature, evidence that the one-body density was not changed significantly. In other experiments there was no evidence of Bose condensation in the momentum distribution. ${ }^{25}$

Day and Beamish ${ }^{26}$ found that there was no static superflow as a result of pressure implying that the phase if it exist is different from a normal superfluid; e. g. a dynamical phenomena. It is also found that there is a strong dependance of the phenomena ${ }^{27}$ on ${ }^{3} \mathrm{He}$ concentration even at the concentration of parts per billion. Parts per million of ${ }^{3} \mathrm{He}$ increase the superfluid response, but have smaller effect on the transition temperature. This dependance is understandable if the ${ }^{3} \mathrm{He}$ goes to lower dimensional structures such as dislocations or grain boundaries.

Rittner and Reppy ${ }^{28}$ have studied how the superfluid density depends on the sample geometry and sample preparation. By making thin samples, they report NCRI up to $20 \%$ much larger than could conceivably generated in even a disordered solid. We believe this shows that the fundamental interpretation of the TO experiments is incorrect. A TO experiment measures the angular momentum response, which in a torus is given by mass flow times velocity. Various estimates imply that the fraction of the mass that could flow is quite small. However, it seems that the flow velocity could be very much greater than imposed on the sample. Critical velocities of superfluid helium in channels ${ }^{4}$ are of order of $1 \mathrm{~m} / \mathrm{sec}$. Hence, a simple model is of a coordinated melting-freezing oscillation, with mass being transported by superfluid through a grain boundary. This oscillation could be tuned to be in resonance with the TO frequency by the motion of ${ }^{3} \mathrm{He}$ impurities and is controlled by the strain fields in the crystal produced by the oscillations. PIMC has been used to look at crystal grain boundaries, ${ }^{29}$ but it is not obvious if such calculations which assume equilibrium, are relevant to the experimental situation.

\section{Acknowledgments}

The work done here was performed in collaboration with B. Bernu, B. Clark, and S. Khairallah with financial support from the NASA program in Fundamental Physics, NSF DMR-03 25939 and the CNRS-UIUC exchange 
program. Computer time was provided by NCSA and the MCC.

\section{References}

1. A. F. Andreev and I. M. Lifshitz, Sov. Phys. JETP 29, 1107 (1969).

2. G. V. Chester Phys. Rev. A 2, 256 (1970).

3. E. Kim and M. H. W. Chan, Science 305, 1941 2004; Nature 427, 225 (2004).

4. S. Balibar and F. Caupin, J. Physics CM, in press (2008).

5. N. Prokof'ev, Adv. Phys. 56, 381 (2007).

6. R. P. Feynman, Phys. Rev. 90, 1116 (1953).

7. E. L. Pollock and D. M. Ceperley, Phys. Rev. B 36, 8343 (1987).

8. D.M. Ceperley and E.L. Pollock, Phys. Rev. Lett. 56, 351 (1986).

9. D. M. Ceperley, Rev. Mod. Phys. 67, 279 (1995).

10. Clark, B. K. and D. M. Ceperley, Path Integral Calculations of Vacancies in Solid Helium, CCP07 conference proceedings, Brussels, Computer Physics Communications, in press (2008).

11. M. Boninsegni, A. B. Kuklov, L. Pollet, N. V. Prokofev, B. V. Svistunov, and M. Troyer, Phys. Rev. Lett. 97, 080401 (2006).

12. D. E. Galli, M. Rossi, and L. Reatto, Phys. Rev. B 71, 140506 (2005).

13. D. E. Galli and L. Reatto, Phys. Rev. Lett. 96, 165301 (2006).

14. N. Prokofev and B. Svistunov, Phys. Rev. Lett. 94, 155302 (2005).

15. B. Clark and D. M. Ceperley, Phys. Rev. Letts. 96,105301:1-4 (2006); condmat/0512547.

16. D. M. Ceperley and B. Bernu, Phys. Rev. Letts. 93, 155303: 1-4 (2004): cond-mat/040933.

17. D. M. Ceperley and G. Jacucci,Phys. Rev. Lett. 58, 1648 (1987).

18. B. Svistunov, talk at Stillwater conference (2007).

19. R. A. Aziz , A. R. Janzen and M. R. Moldover, Phys. Rev. Lett. 74, 1586 (1995).

20. E. W. Draeger and D. M. Ceperley, Phys. Rev. B61, 12094 (2000).

21. E. Vitali, M. Rossi, F. Tramonto, D. E. Galli, and L. Reatto, preprint arXiv:0801.1753 (2008).

22. B. Bernu, and D. M. Ceperley, J. of Phys.and Chem. of Solids, 66, 1462 (2005) cond-mat/0502486.

23. X. Lin, A. C. Clark and M. H. W. Chan, Nature 449, 1025 (2007).

24. E. Blackburn et. al. Phys. Rev. B 76, 024523 (2007);Adams M A, et al. Phys. Rev. Lett. 98085301 (2007).

25. S. O. Diallo et al, Phys Rev. Lett. 98205301 (2007).

26. J. Day and J. Beamish, Phys. Rev. Lett. 96, 105304 (2006)

27. A. C. Clark, J. T. West and M. H. W. Chan, Phys. Rev. Lett. 99135302 (2007).

28. A. S. Rittner and J. D. Reppy, Phys. Rev. Lett. 97, 165301 (2006); 98, 175302 (2007).

29. E. Burovski et al. Phys. Rev. Lett. 94 165301(2005); L. Pollet et al., Phys. Rev. Lett. 98135301 (2007). 